Rabaska

Revue d'ethnologie de l'Amérique française

\title{
Marie-Louise Tenèze (1922-2016)
}

\section{Josiane Bru}

Volume 15, 2017

URI : https://id.erudit.org/iderudit/1041131ar

DOI : https://doi.org/10.7202/1041131ar

Aller au sommaire du numéro

Éditeur(s)

Société québécoise d'ethnologie

ISSN

1703-7433 (imprimé)

1916-7350 (numérique)

Découvrir la revue

Citer ce document

Bru, J. (2017). Marie-Louise Tenèze (1922-2016). Rabaska, 15, 204-206.

https://doi.org/10.7202/1041131ar

Ce document est protégé par la loi sur le droit d'auteur. L'utilisation des services d'Érudit (y compris la reproduction) est assujettie à sa politique d'utilisation que vous pouvez consulter en ligne.

https://apropos.erudit.org/fr/usagers/politique-dutilisation/
Cet article est diffusé et préservé par Érudit.

Érudit est un consortium interuniversitaire sans but lucratif composé de l’Université de Montréal, l'Université Laval et l'Université du Québec à Montréal. Il a pour mission la promotion et la valorisation de la recherche. https://www.erudit.org/fr/ 


\section{Marie-Louise Tenèze (1922-2016)}

Marie-Louise Tenèze est née en 1922 à Longeville-lès-St-Avold, dans le département de la Moselle. Elle est décédée à Paris le 12 octobre 2016, à l'âge de 94 ans. Son ouvre la plus connue est Le Conte populaire français : catalogue raisonné des versions de France et des pays de langue française d'outre-mer, une entreprise initialement conduite par Paul Delarue et laissée inachevée par celui-ci. Elle a été en relation avec la plupart des chercheurs de l'Amérique française qui se sont intéressés au conte populaire, à commencer par Luc Lacourcière et ses collaborateurs.

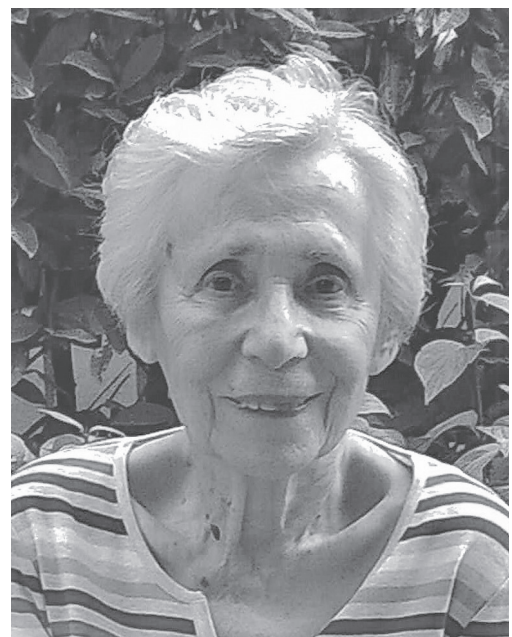

Photo : gracieuseté de la famille Tenèze

Après des études de germanistique, romanistique et folklore à l'Université de Heidelberg et la rédaction d'un mémoire sur les Coutumes et croyances lorraines concernant le feu et l'eau, Marie-Louise Tenèze entreprend une licence de Lettres modernes à l'Université de Strasbourg. Stagiaire du CNRS à l'Institut des hautes études alsaciennes, elle travaille alors au recensement des travaux d'ethnologie régionale et à la diffusion du questionnaire d'enquête en vue de l'Atlas folklorique de France sur cette région.

Entrée au Centre d'ethnographie française (plus tard Centre d'ethnologie française, CNRs et Musée national des arts et traditions populaires) à Paris, elle prend en charge le bulletin Le Mois d'ethnographie française puis la revue Arts et Traditions populaires et rédige la partie française de la Bibliographie internationale des arts et traditions populaires.

La rencontre de Paul Delarue, qui termine le premier volume de son Catalogue raisonné des contes populaires français, marque le tournant décisif dans son itinéraire. Après la mort de celui-ci, en 1956, elle assure la 
continuation du catalogue, communément dénommé dès lors « Catalogue Delarue-Tenèze $»$.

Suivant le même mode de présentation des contes et des versions, elle publie en 1964 le second volume traitant des contes merveilleux ${ }^{1}$, ainsi que l'a conçu Delarue : un catalogue " raisonné », analytique, très détaillé, des " versions de France et des pays de langue française d'outre-mer ", mais elle imprime sa propre marque aux volumes suivants. Les volumes « Contes d'animaux » en $1976^{2}$ et « Contes religieux » en $1985^{3}$, s'ouvrent sur une étude introductive visant à caractériser le genre et à le délimiter par rapport aux récits proches ou encore à le mettre en relation avec d'autres formes de la littérature orale traitant des mêmes sujets. Tenèze insiste sur la proximité qu'entretiennent certains contes-types, qu'elle analyse ensemble, quelle que soit leur place (ou leur absence de place) dans l'Aarne-Thompson. Elle affirme la nécessité d'envisager les variations régionales des contes-types tout en les rattachant, à l'opposé, aux grands ensembles culturels dont l' « entité France » est le point de rencontre. Elle fait paraître, en 2000, le volume concernant les Contes-nouvelles ${ }^{4}$.

Ayant terminé la partie concernant les contes merveilleux et avant d'aborder la suite du chantier, elle passe de l'archive au terrain en prenant la responsabilité de l'enquête de littérature orale dans le cadre de la Recherche coopérative sur programme du CNRS en Aubrac, au sud du Massif central. Elle y travaille avec Alain Rudelle, chargé de la partie linguistique occitane, et noue un dialogue fructueux avec Jean-Michel Guilcher, qui y conduit l'enquête sur les danses populaires. Au contact des conteurs et conteuses, porteurs actifs de la tradition, elle met en évidence les processus de production et de transmission des récits oraux et leurs liens avec les autres aspects de la culture régionale.

Elle poursuivra cette approche du conteur « en acte » en explorant les archives du folkloriste Victor Smith qui, au milieu du XIX ${ }^{\mathrm{e}}$ siècle, s'était attaché à la personnalité et au répertoire d'une fabuleuse conteuse et chanteuse, Nannette Lévesque. Ce répertoire restait inédit malgré le souci qu'en avait eu Paul Delarue. Insistant sur sa forte cohérence, liée à la vision du monde de la

1. Paul Delarue $e^{\dagger}$ et Marie-Louise Tenèze, Le Conte populaire français. Catalogue raisonné des versions de France... Tome deuxième [Contes merveilleux, deuxième partie]., Paris, G.-P. Maisonneuve et Larose, 1964.

2. Marie-Louise Tenèze, Le Conte populaire français. Catalogue raisonné des versions de France... Tome troisième [Contes d'animaux], Paris, G.-P. Maisonneuve et Larose, 1976.

3. Marie-Louise Tenèze, Le Conte populaire français. Catalogue raisonné des versions de France... Tome quatrième, premier volume [Contes religieux], Paris, G.-P. Maisonneuve et Larose, 1985. Une réimpression en un seul volume est parue en 1997 chez le même éditeur.

4. Marie-Louise Tenèze (avec la collaboration de Josiane Bru), Le Conte populaire français : Contes-nouvelles. Catalogue raisonné des versions de France et des pays de langue française d'outremer, Tome quatrième, deuxième volume. Paris, Éd. du Cтнs, « Références de l'ethnologie », 2000. 
conteuse, Marie-Louise Tenèze publie et analyse ce répertoire complet ainsi que les nombreuses notes de Smith, cependant que Georges Delarue édite les chansons dans le même ouvrage, paru en 2000 aux éditions Gallimard5. Les contes du Velay recueillis par Smith auprès d'autres conteurs font l'objet d'un second ouvrage en $2005^{6}$.

La recherche de Marie-Louise Tenèze sur les contes merveilleux comme genre aboutit en 2004 à la publication d'un ouvrage théorique sur Les Contes merveilleux français, recherche de leurs organisations narratives ${ }^{7}$. Elle accepte comme hypothèse de départ la conclusion de Propp sur l'unité morphologique de ce genre narratif, mais s'appuie également sur la notion de conte-type, définie en 1910 par le folkloriste finnois Antti Aarne, qui sert de base à la classification internationale des contes populaires et donc au catalogue français. M.-L. Tenèze propose de mettre à jour un ordre interne de ce genre narratif particulier qu'est le conte merveilleux. Elle y distingue des groupes et sous-groupes sur la base de la série de « choix » distinctifs, qu'ils réalisent. En conclusion, prenant ses distances par rapport à l'affirmation proppienne d'une forme archétypale pour tous les contes merveilleux, elle avance l'hypothèse d'une évolution de la composition et de la signification du genre.

Membre du bureau de l'IsfNR dès sa fondation, de 1960 à 1964, MarieLouise Tenèze a été, à partir de 1992, membre d'honneur des Folklore Fellows à Helsinki. Tout au long de sa longue carrière, elle s'est attachée autant à comprendre les processus de composition et de transmission des récits populaires qu'à la question de leur classement. Ses articles la montrent soucieuse de replacer les contes dans leurs « contextes », ouverte aussi au dialogue avec d'autres disciplines comme la sociologie de la famille, la psychologie ou les études d'ethno-littérature.

Le travail de Marie-Louise Tenèze concernant le catalogue des contes populaires français continue à Toulouse, au Lisst/Centre d'anthropologie sociale : un volume de supplément au catalogue des contes merveilleux français paraîtra aux Presses universitaires du Midi en 2017. La transcription des contes recueillis en occitan en Aubrac lors de sa mission CNRS de 1964-1966 est en cours en vue d'une publication aux éditions Letras d'Oc (avec accord de l'auteur de la collecte).

JoSIANE BRU LissT/Centre d'anthropologie sociale, Toulouse

5. Nannette Lévesque, conteuse et chanteuse du pays des sources de la Loire, Édition établie par Marie-Louise Tenèze et Georges Delarue, Paris, Gallimard, «Le langage des contes », 2000.

6. Contes du Velay. Contes recueillis par Victor Smith de 1869 à 1876, Commentaires de MarieLouise Tenèze, Retournac [Haute-Loire], Éditions du Musée des manufactures de dentelles, 2005.

7. Marie-Louise Tenèze, Les Contes merveilleux français. Recherche de leurs organisations narratives, Paris, Maisonneuve et Larose, 2004. 